\title{
Multidisciplinary Optimization of Urban-Air-Mobility Class Aircraft Trajectories with Acoustic Constraints
}

\author{
Robert D. Falck* and Daniel Ingraham ${ }^{\dagger}$ and Eliot Aretskin-Hariton* \\ NASA John H. Glenn Research Center, Cleveland, OH, 44135
}

The design and analysis of on-demand mobility class vehicles will require thorough acoustic analysis to ensure that more numerous aircraft can operate in densely populated areas without causing excessive levels of noise. This work is a step towards a comprehensive ODM vehicle analysis capability. The authors use 6DOF equations of motion to model the electric quadrotor concept developed by NASA's Revolutionary Vertical Lift Technologies program. As a first step towards acoustic analysis, this trajectory is coupled to an acoustic model that tracks the sound pressure level perceived by an acoustic observer on the ground. The results show that the approach is successful in finding trajectories that minimize total propulsive impulse while obeying limits imposed on the sound pressure level. Future work will involve adding acoustic analysis of increasing fidelity and tying the resulting trajectories to the performance of the electric propulsion system.

\section{Nomenclature}

$=$ body frame

$=$ inertial (Earth-fixed) frame

$=$ north position component along $\hat{e}_{1}$

$=$ east position component along $\hat{e}_{2}$

$=$ altitude (position along $-\hat{e}_{3}$ )

$=$ body-relative velocity component along $\hat{b}_{1}$

$=$ body-relative velocity component along $\hat{b}_{2}$

$=$ body-relative velocity component along $\hat{b}_{3}$

$=$ first rotation, about $\hat{e}_{3}$, for inertial-to-body transformation

$=$ second rotation, about $\hat{e}_{2}^{\prime}$, for inertial-to-body transformation

$=$ third rotation, about $\hat{e}_{1}^{\prime \prime}$, for inertial-to-body transformation

$=$ roll rate about $\hat{b}_{1}$

$=$ pitch rate about $\hat{b}_{2}$

$=$ yaw rate about $\hat{b}_{3}$

$T_{z} \quad=$ total thrust component in the $\hat{b}_{3}$ axis.

$M_{x} \quad=$ roll moment due to thrust differential about $\hat{b}_{1}$ axis.

$M_{y} \quad=$ pitch moment due to thrust differential about $\hat{b}_{2}$ axis.

$M_{z} \quad=$ yaw moment due to propeller moment differential about about $\hat{b}_{3}$ axis.

$p^{\prime} \quad=$ acoustic pressure

$Q \quad=$ monopole source strength

$\vec{x}=$ acoustic observer position vector

$\vec{y}=$ monopole source position vector $\left[p_{n}, p_{e},-h\right]$

$r \quad=$ distance from the source (vehicle) to the observer

$r_{\text {keepout }}=$ distance from the center of the cylindrical geographical constraint

$c_{0} \quad=$ ambient speed of sound

$\omega_{0}=$ source frequency in the inertial frame

$\omega=$ source frequency in acoustic observer's frame

\footnotetext{
*Aerospace Engineer, Mission Architecture and Design Branch, robert.d.falck@nasa.gov, AIAA Member.

†Aerospace Engineer, Acoustics Branch, daniel.j.ingraham@nasa.gov, AIAA Member.

$\ddagger$ Aerospace Technologist Control Engineer, Intelligent Control and Autonomy Branch, eliot.d.aretskin-hariton@nasa.gov.
} 
$V_{\text {inertial }}=$ source (vehicle) velocity in inertial frame

$M_{r} \quad=$ source velocity in direction of the observer divided by $c_{0}$

$S P L=$ sound pressure level

$p_{\text {ref }} \quad=$ reference pressure for SPL calculation

\section{Introduction}

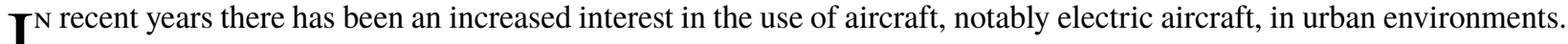
So-called "on-demand-mobility" (ODM) and "thin-haul" applications of aircraft would place aircraft in closer proximity to people by operating from more numerous locations. Thin-haul applications would more heavily utilize existing airports that are currently operating below capacity, while ODM applications are more typically thought of as "air-taxis" operating in and around urban environments. This close-proximity of aircraft with respect to populated areas during takeoff and landing raises questions about noise levels and how they might be mitigated.

In this work the authors demonstrate an approach to noise-mitigation that involves both aircraft trajectory and propulsion-airframe design and integration. Optimal control is performed using Dymos, an optimal-control library built on top of NASA's OpenMDAO framework [1]. The Dymos library allows us to efficiently solve optimal control problems using well-defined techniques like Gauss-Lobatto collocation [2] or the Radau Pseudospectral Method [3]. Dymos leverages the modularity of OpenMDAO to provide general trajectory optimization capability using a wide array of possible constraints and objective functions coming from several different disciplinary subsystem models. A key feature of this setup is the propagation of analytic derivatives throughout the entire model which enables more robust convergence of the resulting nonlinear programming problem through the use of gradient-based optimization techniques.

Previous work on trajectory optimization with acoustic constraints was conducted by Tsuchiya, Ishii, et al. who used a response surface model for a noise source model in conjunction with analytic noise attenuation models to optimize final approach trajectories for helicopters by minimizing a combination of velocity change and time-averaged noise estimates [4]. In addition, Atkins and Xue demonstrated trajectory optimization for final approach of "runwayindependent" (VTOL) aircraft which used a combination of fuel burn, transit time, and radiated noise level as the objective function [5]. In this work, rather than trying to find an appropriate combination of factors for the objective we stick to a single performance metric, total impulse, while imposing constraints on time-of-flight and maximum noise levels. This allows us to more easily perform trade studies which demonstrate the sensitivity of aircraft performance to various constraints.

\section{System Modeling}

\section{A. Equations of Motion}

For the purposes of this paper, the aircraft modeled is a battery-powered vertical take-off and landing (VTOL) vehicle. The trajectory is modeled using 6DOF equations of motion in order to capture the propeller and motor performance in each nacelle. The equation of motion parameterization is the same as that developed by Beard [6]. The states for the dynamics are as follows:

$$
\bar{x}=\left[\begin{array}{llllllllllll}
p_{n} & p_{e} & h & u & v & w & \phi & \theta & \psi & p & q & r
\end{array}\right]^{T}
$$

The summarized equations of motion as as follows:

$$
\begin{aligned}
& {\left[\begin{array}{c}
\dot{p_{n}} \\
\dot{p_{e}} \\
\dot{h}
\end{array}\right]=\left[\begin{array}{ccc}
c_{\theta} c_{\psi} & s_{\phi} s_{\theta} c_{\psi}-c_{\phi} s_{\psi} & c_{\phi} s_{\theta} c_{\psi}+s_{\phi} s_{\psi} \\
c_{\theta} s_{\psi} & s_{\phi} s_{\theta} s_{\psi}+c_{\phi} c_{\psi} & c_{\phi} s_{\theta} c_{\psi}+s_{\phi} s_{\psi} \\
s_{\theta} & -s \phi c_{\theta} & -c_{\phi} c_{\theta}
\end{array}\right]\left[\begin{array}{c}
u \\
v \\
w
\end{array}\right]} \\
& {\left[\begin{array}{c}
\dot{u} \\
\dot{v} \\
\dot{w}
\end{array}\right]=\left[\begin{array}{c}
r v-q w \\
p w-r u \\
q u-p v
\end{array}\right]+\frac{1}{m}\left[\begin{array}{l}
f_{x} \\
f_{y} \\
f_{z}
\end{array}\right]}
\end{aligned}
$$




$$
\begin{aligned}
& {\left[\begin{array}{c}
\dot{\phi} \\
\dot{\theta} \\
\dot{\psi}
\end{array}\right]=\left[\begin{array}{ccc}
1 & s_{\phi} t_{\theta} & c_{\phi} t_{\theta} \\
0 & c_{\phi} & -s_{\phi} \\
0 & \frac{s_{\phi}}{c_{\theta}} & \frac{c_{\phi}}{c_{\theta}}
\end{array}\right]\left[\begin{array}{l}
p \\
q \\
r
\end{array}\right]} \\
& {\left[\begin{array}{c}
\dot{p} \\
\dot{q} \\
\dot{r}
\end{array}\right]=\left[\begin{array}{l}
\frac{J_{y}-J_{z}}{J_{x}} q r \\
\frac{J_{z}-J_{x}}{J_{y}} p r \\
\frac{J_{x}-J_{y}}{J_{z}} p q
\end{array}\right]+\left[\begin{array}{c}
\frac{M_{x}}{J_{x}} \\
\frac{M_{y}}{J_{y}} \\
\frac{M_{z}}{J_{z}}
\end{array}\right]}
\end{aligned}
$$

For a multi-rotor type vehicle, we use a control parameterization that requires four values: the total thrust produced by the rotors, the moment due to thrust-offset about the roll axis, the moment due to thrust-offset the pitch axis, and the total net yaw-moment caused by different shaft torques of the counter-rotating propellers [6,7]. This simplified parameterization reduces the number of design variables in the transcribed nonlinear programming problem.

$$
\bar{u}=\left[\begin{array}{llll}
F_{z} & M_{x} & M_{y} & M_{z}
\end{array}\right]^{T}
$$

\section{B. Vehicle Model}

The vehicle for this analysis is based on the quad-rotor concept developed by NASA's Revolutionary Vertical Lift Technologies (RVLT) program[8]. The vehicle has a gross mass of $567.9 \mathrm{~kg}$. Due to the lack of detailed mass allocation data, the inertia tensor is currently approximated as a sphere with principle moments of inertia of $200 \mathrm{~kg} / \mathrm{m}^{2}$. The maximum total thrust is $6920 \mathrm{~N}$ ( $1730 \mathrm{~N}$ per rotor). The rudimentary aerodynamics model prescribes a drag coefficient of 0.5 and a lift coefficient of zero. Vehicle dimensions are currently inconsequential, but future work will involve coupling the trajectory performance to the propulsion system behavior. Given the control parameterization used in (6), the moment arm of each rotor is need to convert the given thrust and moment values to individual motor power and speed.

\section{Acoustic Model}

The acoustic source selected in this work is a single acoustic monopole,

$$
p^{\prime}\left(\vec{x}, t+\frac{|\vec{x}-\vec{y}|}{c_{0}}\right)=\frac{Q(t)}{4 \pi|\vec{x}-\vec{y}(t)|\left[1-M_{r}(t)\right]}
$$

where $p^{\prime}$ is the acoustic pressure, $Q$ is the monopole source strength, $c_{0}$ is the ambient speed of sound, and $M_{r}=V_{\text {inertial }} / c_{0}$. In (7), $|\vec{x}-\vec{y}(t)|$ is the distance from the monopole source location $\vec{y}$ (in this work, the position of the vehicle) to the "acoustic observer" $\vec{x}$, i.e., the point in space where the noise will be evaluated. Because the vehicle is moving, $\vec{y}$ is a function of time- the observer location $\vec{x}$ is taken to be constant, however.

The monopole source strength in (7) takes the form

$$
Q=Q_{s} \cos \left(\omega_{0} t\right),
$$

with $Q_{s}=2 \pi$ Pa m and $\omega_{0}=100 \mathrm{~Hz}$. Since (8) contains just one frequency $\omega$, the pressure disturbance can be expressed in frequency space as

$$
p^{\prime}(\vec{x}, \omega(t))=\frac{Q(\omega)}{4 \pi|\vec{x}-\vec{y}(t)|\left[1-M_{r}(t)\right]}
$$

where

$$
\omega(t)=\left[1+M_{r}(t)\right] \omega_{0}
$$

accounts for the Doppler shift associated with the relative motion of the source with respect to the acoustic observer.

The acoustic metric chosen for this work is the Sound Pressure Level, which, for the simple source described by (9), is

$$
\mathrm{SPL}=10 \log _{10}\left(\left[\frac{p^{\prime}}{p_{\text {ref }}}\right]^{2}\right)
$$

where $p_{\text {ref }}=20 \mu \mathrm{Pa}$. 


\section{Problem Definition}

This trajectory optimization problem is intended to be a representative trajectory of an UAM-class quadrotor transporting a single passenger (per the RVLT design) or the equivalent weight in cargo over a relatively short distance subject to both a geographic constraint and an acoustic constraint. The vehicle begins at a reference position of $(0 \mathrm{~km}, 0$ $\mathrm{km}, 0 \mathrm{~m})$ in the North-East-Down frame, and must end at $(7 \mathrm{~km}, 7 \mathrm{~km}, 0 \mathrm{~m})$.

Table 1 Bounds for the initial and final values of the states.

\begin{tabular}{lll}
\hline State Variable & Initial Value & Final Value \\
\hline$p_{n}$ & $0.0 \mathrm{~km}$ & $7.0 \mathrm{~km}$ \\
$p_{e}$ & $0.0 \mathrm{~km}$ & $7.0 \mathrm{~km}$ \\
$h$ & $0.0 \mathrm{~m}$ & $0.0 \mathrm{~m}$ \\
$u$ & $0.0 \mathrm{~m} / \mathrm{s}$ & $0.0 \mathrm{~m} / \mathrm{s}$ \\
$v$ & $0.0 \mathrm{~m} / \mathrm{s}$ & $0.0 \mathrm{~m} / \mathrm{s}$ \\
$w$ & $0.0 \mathrm{~m} / \mathrm{s}$ & $0.0 \mathrm{~m} / \mathrm{s}$
\end{tabular}

\begin{tabular}{lll}
\hline State Variable & Initial Value & Final Value \\
\hline$\phi$ & $0.0 \mathrm{rad} / \mathrm{s}$ & $0.0 \mathrm{rad} / \mathrm{s}$ \\
$\theta$ & $0.0 \mathrm{rad} / \mathrm{s}$ & $0.0 \mathrm{rad} / \mathrm{s}$ \\
$\psi$ & $0.0 \mathrm{rad} / \mathrm{s}$ & $0.0 \mathrm{rad} / \mathrm{s}$ \\
$p$ & $0.0 \mathrm{rad} / \mathrm{s}$ & free \\
$q$ & $0.0 \mathrm{rad} / \mathrm{s}$ & free \\
$r$ & $0.0 \mathrm{rad} / \mathrm{s}$ & free \\
$J$ & $0.0 \mathrm{Ns}$ & free
\end{tabular}

To optimize the trajectory, the total impulse provided by the propulsion system is minimized. Since the thrust is entirely in the $-\hat{b}_{3}$ axis, this is computed as:

$$
J=\int_{t_{0}}^{t_{f}}-F_{z} d t
$$

The trajectory is subject to several path constraints. First, the total thrust is bounded limited to the maximum total thrust of the vehicle. A lower bound of $1000 \mathrm{~N}$ is imposed to prevent excessive downward velocity. The pitch and roll moments are constrained to $\pm 5 \mathrm{Nm}$, with rates of $\pm 1 \mathrm{Nm} / \mathrm{s}$. A geographical keepout zone is included to ensure the aircraft can avoid obstacles. This keepout zone is modeled as a cylinder centered at $1 \mathrm{~km}$ North, $2 \mathrm{~km}$ East. The acoustic observer is located on the ground at $4 \mathrm{~km}$ North, $5 \mathrm{~km}$ East. First, an optimization is run with an unconstrained sound pressure level at the observer location. Once the baseline SPL is known, several trajectories were optimized with increasingly tighter limits on the maximum allowable SPL. The constraints are summarized in Table 2.

Table 2 Path constraints

\begin{tabular}{lll}
\hline Path Constraint & Lower bound & Upper bound \\
\hline$F_{z}$ & $1000 \mathrm{~N}$ & $6920.0 \mathrm{~N}$ \\
$\dot{F}_{z}$ & $-300 \mathrm{~N} / \mathrm{s}$ & $300 \mathrm{~N} / \mathrm{s}$ \\
$M_{x}$ & $-5.0 \mathrm{Nm}$ & $5.0 \mathrm{Nm}$ \\
$M_{y}$ & $-5.0 \mathrm{Nm}$ & $5.0 \mathrm{Nm}$ \\
$\dot{M}_{x}$ & $-1.0 \mathrm{Nm} / \mathrm{s}$ & $1.0 \mathrm{Nm} / \mathrm{s}$ \\
$\dot{M}_{y}$ & $-1.0 \mathrm{Nm} / \mathrm{s}$ & $1.0 \mathrm{Nm} / \mathrm{s}$ \\
$r_{\text {keepout }}$ & $1 \mathrm{~km}$ & None \\
$S P L$ & None & varies
\end{tabular}

\section{Results}

Optimizing the trajectory with an unconstrained sound pressure level resulted in a peak SPL of about $27.5 \mathrm{~dB}$. From there, six more cases were run with increasingly strict limits on the maximum SPL: $24 \mathrm{~dB}, 22 \mathrm{~dB}, 20 \mathrm{~dB}$, and $18 \mathrm{~dB}$. Figure 1 shows the flight path taken by the vehicle for varying SPL constraints. The most tightly-constrained case flies further from the acoustic observer in the horizontal plane, but in general there is relatively little variability between the cases from a bird's eye view. 


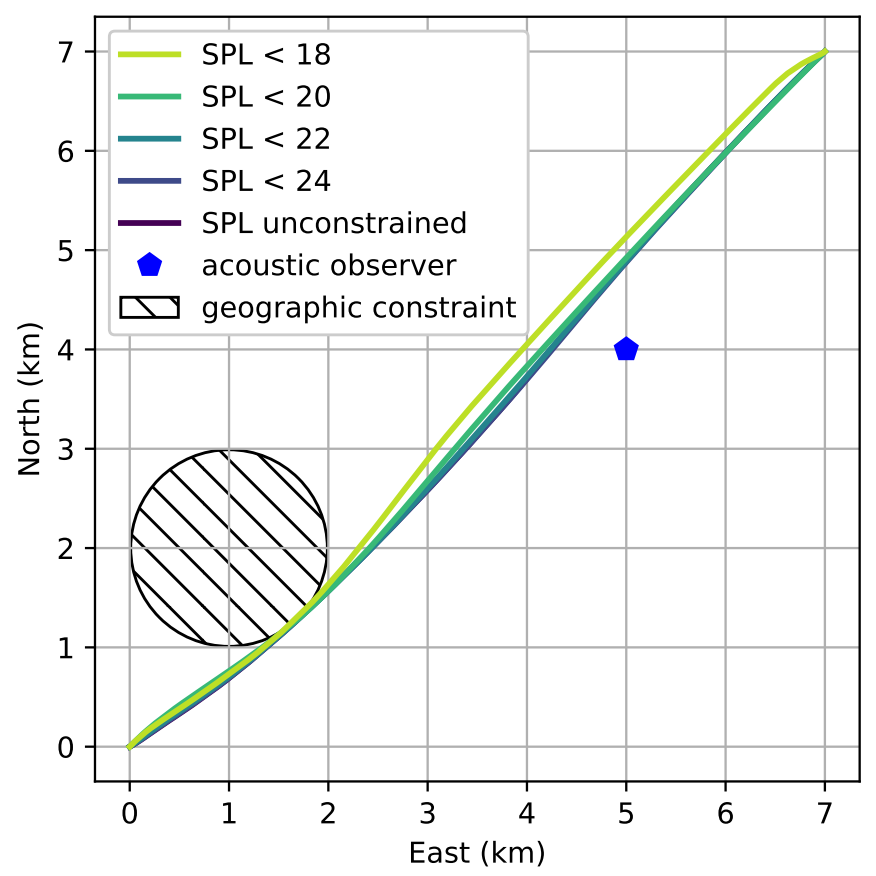

Fig. 1 A top-down view of the flight paths assumed for cases with varying SPL limits. The apparent minor violations of the geographic constraint are due to the discretized nature of the collocation technique.

Figure 2 shows the altitude time-history of each case and gives further insight into way in which the trajectory optimizer satisfies the acoustic constraint. Since this work considered a fixed monopole source strength, the most effective way to achieve a lower SPL is to move farther away from the observer. Rather than moving laterally to avoid the gain larger distance the vehicle flies higher. Doing so increases the distance to the observer, but also increases duration of the flight which increases the total impulse needed to complete the flight.



Fig. 2 The altitude time-history for each trajectory. There is significant variation in the altitude profile as a function of the SPL limit. 
Figure 3 shows the sound pressure level as a function of time for each trajectory. As the SPL constraint becomes more severely limited, the trajectories begin to fly a flight profile that rides the constraint. This changes the fundamental character of the SPL, from having a clear peak around 80 seconds to having more of a constant value from about 65 seconds to 110 seconds.

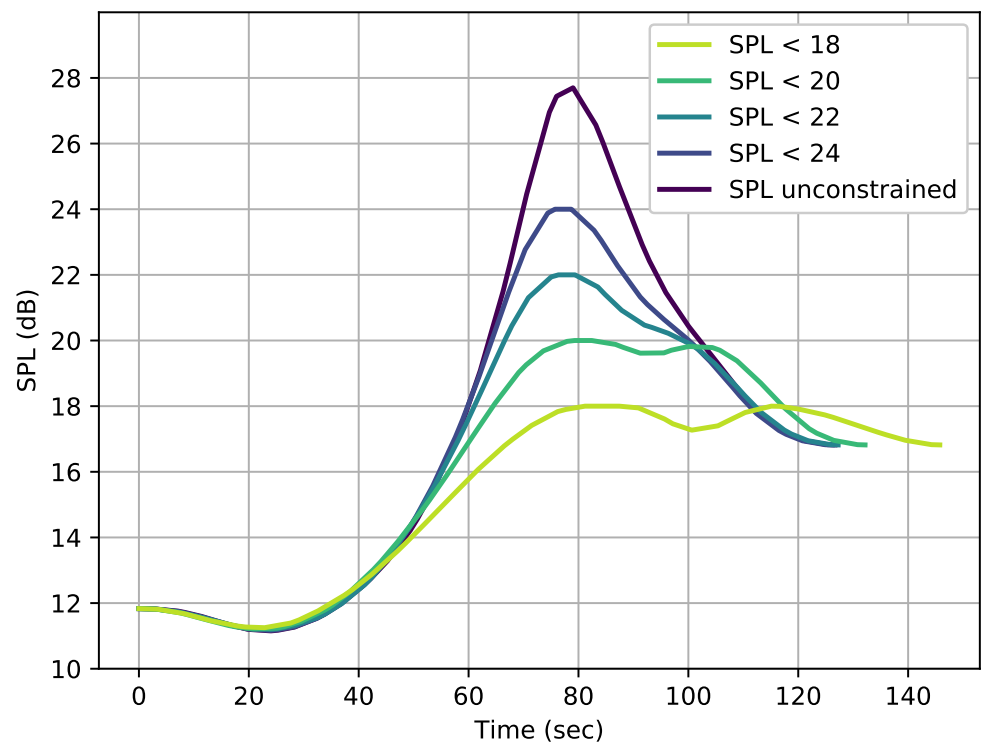

Fig. 3 The sound pressure time-history for each trajectory.

A Pareto front of total impulse vs maximum SPL is plotted Figure 4. The asymptotic behavior at the lower limit of the SPL range is due to the infeasible nature of the problem at excessively low SPL limits. When the vehicle reaches its prescribed final location the SPL is about 17. Again, noting that the monopole has a fixed strength in this work, there is no way for the optimizer to respect the final position and reduce SPL below that level.

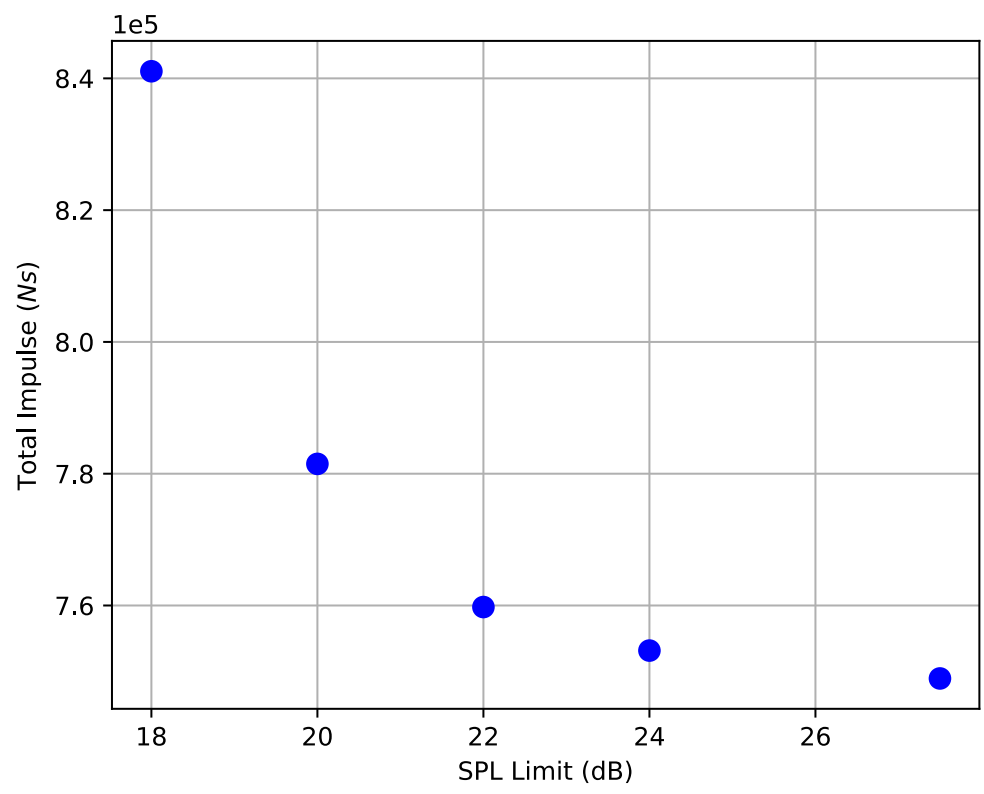

Fig. 4 The total propulsive impulse vs. the sound pressure level limit for each trajectory. Feasible trajectories exist in the region above the Pareto front. 


\section{Conclusions and Future Work}

In this work the authors have demonstrated the ability to incorporate acoustic analysis in a 6DOF trajectory optimization using gradient based optimization with analytic derivatives. In this case aircraft is acoustically modeled as a simple monopole emitting a single frequency and strength that is independent of vehicle design and operating conditions. The optimization was run for a set of different values for the maximum allowable SPL, and the results showed that the most efficient way to respect the constraint was to gain altitude rather than move laterally away from the acoustic observer.

The next steps in this work will involve developing a realistic source noise model that couples to a rotor design analysis. In their works, Beard and ElKholy used simple fixed pitch rotors and were able to derive the rotational speed based on the necessary thrusts, which are derived from the definition of the control vector [6,7]. Large scale quad-rotor vehicles will have more complex drives with variable pitch, and thus will require a more complex model from which the rotational speed and power will be computed. The noise will be modeled to higher fidelity using the Aircraft NOise Prediction Program 2 (ANOPP2), a acoustic prediction framework developed at the NASA Langley Research Center capable of predicting noise from a wide range of aircraft designs, incorporating data from various sources and levels of fidelity[9]. The near-field rotor noise will be estimated using a combination of ANOPP2 and the propeller performance model developed by Hwang and Ning [10]. This will give the optimizer additional degrees of freedom to respect noise constraints, because it will be able to vary throttle setting, blade RPM, and in addition to vehicle location and speed.

\section{Acknowledgments}

The authors thank NASA's Transformative Tools and Technologies program for funding this work.

\section{References}

[1] Gray, J., Moore, K. T., and Naylor, B. A., "OpenMDAO: An open source framework for multidisciplinary analysis and optimization," 13th AIAA/ISSMO Multidisciplinary Analysis and Optimization Conference, Fort Worth, TX, AIAA, AIAA-20109101, 2010, pp. 5-7.

[2] Herman, A. L., and Conway, B. A., "Direct optimization using collocation based on high-order Gauss-Lobatto quadrature rules," Journal of Guidance, Control, and Dynamics, Vol. 19, No. 3, 1996, pp. 592-599.

[3] Garg, D., Patterson, M., Darby, C., Francolin, C., Huntington, G., Hager, W., and Rao, A., Direct Trajectory Optimization and Costate Estimation of General Optimal Control Problems Using a Radau Pseudospectral Method, American Institute of Aeronautics and Astronautics, 2009.

[4] Tsuchiya, T., Ishii, H., Uchida, J., Ikaida, H., Gomi, H., Matayoshi, N., and Okuno, Y., "Flight Trajectory Optimization to Minimize Ground Noise in Helicopter Landing Approach,” Journal of Guidance, Control, and Dynamics, Vol. 32, No. 2, 2009, pp. 605-615. doi:10.2514/1.34458, URL https://doi .org/10.2514/1.34458.

[5] Atkins, E. M., and Xue, M., "Noise-Sensitive Final Approach Trajectory Optimization for Runway-Independent Aircraft," Journal of Aerospace Computing, Information, and Communication, Vol. 1, No. 7, 2004, pp. 269-287. doi:10.2514/1.3924, URL https://doi .org/10.2514/1.3924.

[6] Beard, R. W., “Quadrotor dynamics and control,” Tech. rep., Brigham Young University, 2008.

[7] ElKhol, H. t. M. N., "Dynamic Modeling and Control of a Quadrotor Using Linear and Nonlinear Approaches," Master's thesis, The American University in Cairo, 2014. URL http://dar.aucegypt.edu/bitstream/handle/10526/3965/ Heba $\left\{_{-}\right\}$ElKholy $\left\{_{-}\right\}$Thesis $\left\{_{-}\right\}$S2014.pdf? sequence $=1$.

[8] Johnson, W., Silva, C., and Solis, E., “Concept Vehicles for VTOL Air Taxi Operations,” AHS International Technical Meeting on Aeromechanics Design for Transformative Vertical Flight, San Francisco, CA, 2018.

[9] Lopes, L., and Burley, C., "Design of the Next Generation Aircraft Noise Prediction Program: ANOPP2," 17th AIAA/CEAS Aeroacoustics Conference (32nd AIAA Aeroacoustics Conference), Aeroacoustics Conferences, American Institute of Aeronautics and Astronautics, 2011. doi:doi:10.2514/6.2011-2854, URL https://doi .org/10.2514/6.2011-2854.

[10] Hwang, J. T., and Ning, A., "Large-scale multidisciplinary optimization of an electric aircraft for on-demand mobility," 2018 AIAA/ASCE/AHS/ASC Structures, Structural Dynamics, and Materials Conference, AIAA SciTech Forum, American Institute of Aeronautics and Astronautics, 2018. doi:doi:10.2514/6.2018-1384, URL https://doi .org/10.2514/6.2018-1384. 